\title{
NECESSARY AND SUFFICIENT OPTIMALITY CONDITIONS FOR D.C VECTOR OPTIMIZATION
}

\author{
A. Shafie
}

ABSTRACT. In this paper, the vector version of the $\phi$-directionally approximate pseudo-dissipativity for set valued mappings is introduced. By this concept, neccessary and sufficient optimally conditions for D.C. vector optimization problems are established. A new proof for the vector version of Farkas Lemma, in the setting of convex-like mapping is presented. Finally some examples are supported the main results.

2010 Mathematics Subject Classification: 46N10,49Kxx.

Keywords: Convex mapping, Optimality condition, Local weak minimal solution, Subdifferential, Proper minimal point.

\section{Preliminaries}

Throughout this paper, $X, Y$ and $Z$ stand for topological vector spaces. Let $Y^{*}$ and $Z^{*}$ be topological duals of $Y$ and $Z$, respectively, and $\langle.,$.$\rangle be the duality pairing.$ Let $K \subset Y$ and $D \subset Z$ be proper $(K \neq \varnothing$ and $K \neq Y)$ convex cones with nonempty interior $($ int $K \neq \emptyset$, int $D \neq \emptyset$ ). The set of all linear continuous functions from $X$ to $Y$ is denoted by $L(X, Y)$ and $l(K)=K \cap(-K)$ is the linearity of $K$. If $K \cap(-K)=\{0\}$, then $K$ is called pointed. Let $Y$ and $Z$ be partially ordered by $K$ and $D$, respectively. The ordering relations induced on $Y$ and $Z$ denoted by $\leq_{K}$ and $\leq_{D}$, respectively, consist of

$$
y_{1} \leq_{K} y_{2} \Longleftrightarrow y_{2} \in y_{1}+K
$$

and

$$
z_{1} \leq_{D} z_{2} \Longleftrightarrow z_{2} \in z_{1}+D
$$

The negative polar cone and the strict negative polar cone $K^{*}$ of $K$ are denoted by $K^{*}$ and $\left(K^{*}\right)^{\circ}$, and defined as follows:

$$
K^{*}=\left\{y^{*} \in Y^{*}:\left\langle y^{*}, y\right\rangle \geq 0, \quad \forall y \in K\right\}
$$


A. Shafie - Necessary and sufficient optimality conditions for ...

and,

$$
\left(K^{*}\right)^{\circ}=\left\{y^{*} \in Y^{*}:\left\langle y^{*}, y\right\rangle>0, \quad \forall y \in K \backslash l(K)\right\} .
$$

The negative polar cone $D^{*}$ of $D$ and the strict negative polar cone $\left(D^{*}\right)^{\circ}$ are defined similarly. It is clear that he negative polar cone and the strict negative polar cone are convex and cone. The indicator function $\delta_{M}: X \longrightarrow \mathbb{R} \cup\{+\infty\}$ of a set $M \subseteq X$ is defined by

$$
\delta_{M}(x)= \begin{cases}0 & \text { if } x \in M, \\ +\infty & \text { if } x \notin M .\end{cases}
$$

Definition 1.1. Let $A$ be a nonempty subset of $Y$. An element $\bar{y} \in A$ is called a Pareto minimal or efficient point of $A$ with respect to $K$ and is denoted by $\bar{y} \in$ MinA, iff

$$
(A-\bar{y}) \cap(-K \backslash\{0\})=\emptyset,
$$

and $\bar{y}$ is said to be a weak Pareto minimal point of $A$ with respect to $K$ and is denoted by $\bar{y} \in \mathrm{WMinA}$ iff

$$
(A-\bar{y}) \cap(-\operatorname{intK})=\emptyset .
$$

Note that $\operatorname{Min} A \subseteq W \operatorname{Min} A$, also the set of all weak Pareto minimal points of $A$ is closed.

Definition 1.2. Let $A$ be a nonempty subset of $Y$ and $\epsilon \in K$. An element $\bar{y} \in A$ is called an $\epsilon$-weak Pareto minimal point of $A$ with respect to $K$ and is denoted by $\bar{y} \in \epsilon-\mathrm{WMinA}$ iff

$$
(A-\bar{y}+\epsilon) \cap(-\operatorname{intK})=\emptyset .
$$

In the similar way we can define $\epsilon$ - Pareto minimal point of a set. It is strightforward to check that $W \operatorname{Min} A \subset \bigcap_{\epsilon \in K} \epsilon-W \operatorname{Min} A$. Since the convexity plays an important role in vector optimization and especially in this paper, we recall it. The mapping $F: X \rightarrow Y$ is said to be $K$-convex, if for every $\alpha \in[0,1]$ and $x, y \in X$

$$
\alpha F(x)+(1-\alpha) F(y) \in F(\alpha x+(1-\alpha) y)+K .
$$

and the mapping $F: X \rightarrow Y$ is said to be $K$-convexlike on $S$ if for any $x, y \in S, \lambda \in$ $[0,1]$ there exists $z \in S$ such that

$$
\lambda F(x)+(1-\lambda) F(y)-F(z) \in K
$$

Remark that if we take $Y=\Re$ and $K=[0, \infty)$ then the $K$ - convexity of $F$ reduces to the usual definition of the convexity for a function. 
A. Shafie - Necessary and sufficient optimality conditions for ...

Definition 1.3. Let $F: X \longrightarrow Y$ be a given map. The subdifferential of $F$ at $\bar{x} \in X$ is given by

$$
\partial F(\bar{x})=\left\{T \in L(X, Y): F(x)-F(\bar{x}) \geq_{K} T(x-\bar{x}) \quad \forall x \in X\right\} .
$$

It is clear that $0 \in \partial F(\bar{x})$ iff $F(x) \geq_{K} F(\bar{x})$ for all $x \in K$. This means $F(\bar{x})$ is Pareto minimal point of the range $F$ iff the zero mapping belongs to the subdifferential of $F$ at $\bar{x}$. Let $\epsilon \in K$. The $\epsilon$-subdifferential of $F$ at $\bar{x} \in X$ is given by

$$
\partial_{\epsilon} F(\bar{x}):=\left\{T \in L(X, Y): F(x)-F(\bar{x})+\epsilon \geq_{K} T(x-\bar{x}) \quad \forall x \in X\right\} .
$$

It is straightforward to check that $\partial_{\epsilon} F(\bar{x})=\bigcap_{\substack{\epsilon \leq \beta \\ \beta \in K}} \partial_{\beta} F(\bar{x})$. Every $T \in \partial_{\epsilon} F(\bar{x})$ is called an $\epsilon$-subgradient. If $y^{*} \in Y^{*}$, then

$$
y^{*} \circ \partial F(\bar{x})=\left\{y^{*} \circ T: T \in \partial F(\bar{x})\right\} .
$$

In this paper, we consider the following cone-constrained vector optimization problem, sometimes called D-C vector optimization where D-C refers to difference of two convex functions:

$$
(P)\left\{\begin{array}{l}
K-\operatorname{Min} F(x)-G(x), \\
\text { subject to } x \in C \text { and } H(x)-S(x) \in-D .
\end{array}\right.
$$

where $F, G: X \longrightarrow Y$ are $K$-convex and $S, H: X \longrightarrow Z$ are $D$-convex maps.

Definition 1.4. [12] Suppose that $\Omega:=\{x \in C: H(x)-S(x) \in-D\}$ and $\epsilon \in K$. An element $\bar{x} \in \Omega$ is called an $\epsilon$-weak local Pareto minimal solution of problem $(P)$ iff there exists a neighborhood $U$ of $\bar{x}$ such that

$$
F(\bar{x})-G(\bar{x}) \in \epsilon-\mathrm{WMin}(G-F)(U \cap \Omega),
$$

i.e.,

$$
(F-G)(U \cap \Omega) \subset F(\bar{x})-G(\bar{x})-\epsilon+Y \backslash-i n t K,
$$

where $(F-G)(U \cap \Omega)=\{F(x)-G(x): x \in U \cap \Omega\}$.

\section{Sufficient Optimality Conditions}

In the sequel, let $X$ be a normed space and $\phi$ be a positive bi-function $\phi: X \times$ $X \longrightarrow \mathbb{R}_{+}$. In what follows, using a generalized notion of monotonicity, we establish new sufficient optimally conditions for an $\epsilon$-weak Pareto minimal solution for the vector optimization problem $(P)$. The following definition extends the notion of directionally approximately pseudo-dissipative in norm space $X$. 
A. Shafie - Necessary and sufficient optimality conditions for ...

Definition 2.1. A set-valued map $M: X \rightrightarrows L(X, Y)$ is called $\phi$-directionally approximately pseudo-dissipative at $\bar{x} \in X$ iff, for every $u \in S_{X}$ and $\epsilon \in i n t K$, there exists $\delta>0$ such that

$\forall x \in B(u, \delta), \forall t \in(0, \delta) \exists T^{*} \in M(\bar{x}+t x), \exists T \in M(\bar{x}):\left(T^{*}-T\right)(x) \leq_{K} \epsilon \phi(x, \bar{x})$.

Note that if $\phi(x, y)=1$, Definition 2.1 reduces to the directionally approximately pseudo-dissipative.

Remark 2.1. Any single valued mapping is $\phi$-directionally approximately pseudodissipative .

Remark 2.2. If $Y=\mathbb{C}$ or $\mathbb{R}$, Definition 2.1 reduces to the definition of a directionally approximate pseudo-dissipativity introduced by Penot [4].

It must be noted that the class of approximately pseudo-dissipative maps is a subset of the class of directionally approximately pseudo-dissipative maps. In fact, every directionally gap-continuous mapping at $\bar{x}$ is directionally approximately pseudo-dissipative at $\bar{x}$.

Theorem 2.1. Let $\bar{x} \in \Omega$. Assume that the set-valued maps $\left(\partial_{\frac{\epsilon}{2}} F\right)$ and $(\partial H)$ are both $\phi$-directionally approximately pseudo-dissipative at $\bar{x}$ and $\phi$ is a bounded bifunction. If , for all $T \in \partial_{\frac{\epsilon}{2}} F(\bar{x})$ and $L \in \partial H(\bar{x})$, there exist $\left(y^{*}, z^{*}\right) \in K^{*} \backslash\{0\} \times D^{*}$ such that

$$
\left\{\begin{array}{l}
y^{*} \circ(-T)+z^{*} \circ(-L) \in \partial\left(y^{*} \circ(-G)+z^{*} \circ(-S)\right)(\bar{x}), \\
\left\langle z^{*}, H(\bar{x})-S(\bar{x})\right\rangle=0
\end{array}\right.
$$

then $\bar{x}$ is an $\epsilon$-weak local Pareto minimal solution of problem $(P)$.

Proof. From $\phi$-directionally approximately pseudo-dissipativity of the set-valued maps $\partial_{\frac{\epsilon}{2}} F$ and $\partial H$ at $\bar{x}$, there exists $\delta>0$ such that for each $t \in(0, \delta)$ and $v \in B(0, \delta)$ we have

$$
\exists T^{\prime} \in \partial_{\frac{\varepsilon}{2}} F(\bar{x}+t v), T \in \partial_{\frac{\epsilon}{2}} F(\bar{x}) \text { such that }\left(T^{\prime}-T\right)_{(v)} \leq_{K} \alpha \phi(\bar{x}, v),
$$

and

$$
\exists L^{\prime} \in \partial H(\bar{x}+t v), L \in \partial H(\bar{x}) \text { such that }\left(L^{\prime}-L\right)_{(v)} \leq_{D} \gamma \phi(\bar{x}, v),
$$

where $\alpha \in \operatorname{int} K, \gamma \in \operatorname{int} D$ and $u=0$ for Definition 2.1. Now, set $U=\bar{x}+B(0, \delta)$. As $x-\bar{x} \in B(0, \delta)$, so for any $x \in U \cap \Omega$, it follows that

$$
\exists T^{\prime} \in \partial_{\frac{\epsilon}{2}} F(\bar{x}+t(\bar{x}-x)), T \in \partial_{\frac{\epsilon}{2}} F(\bar{x}) \text { such that }\left(T-T^{\prime}\right)(x-\bar{x}) \leq_{K} \alpha \phi(\bar{x}, x-\bar{x}),
$$


and

$$
\exists L^{\prime} \in \partial H(\bar{x}+t(\bar{x}-x)), L \in \partial H(\bar{x}) \text { such that }\left(L-L^{\prime}\right)(x-\bar{x}) \leq_{D} \gamma \phi(\bar{x}, x-\bar{x}) .
$$

Since $T^{\prime} \in \partial_{\frac{\epsilon}{2}} F(\bar{x}+t(x-\bar{x}))$ and $T \in \partial_{\frac{\epsilon}{2}} F(\bar{x})$, we have

$$
F(y)-F(\bar{x}+t(\bar{x}-x))-T^{\prime}(y-(\bar{x}+t(\bar{x}-x)))+\frac{\epsilon}{2} \in K \quad \forall y \in X,
$$

and,

$$
F(y)-F(\bar{x})-T(y-\bar{x})+\frac{\epsilon}{2} \in K \quad \forall y \in X .
$$

For the case $y=x$ in (4) and $y=\bar{x}+t(\bar{x}-x)$ in (5) we have,

$$
F(x)-F(\bar{x}+t(\bar{x}-x))-(1+t) T^{\prime}(x-\bar{x})+\frac{\epsilon}{2} \in K,
$$

and,

$$
F(\bar{x}+t(\bar{x}-x))-F(\bar{x})+t T(x-\bar{x})+\frac{\epsilon}{2} \in K .
$$

By adding (6) to (7), we get

$$
F(x)-F(\bar{x})-\left[-t T+(1+t) T^{\prime}\right](x-\bar{x})+\epsilon \in K .
$$

By using the same argument and $L^{\prime} \in \partial H(\bar{x}+t(\bar{x}-x)$ and $L \in \partial H(\bar{x})$ we have

$$
H(x)-H(\bar{x})-\left[-t L+(1+t) L^{\prime}\right](x-\bar{x}) \in D .
$$

Thus, for every $y_{1}^{*} \in K^{*}$ and $z_{1}^{*} \in D^{*}$ we have,

$$
\begin{aligned}
& \left\langle y_{1}^{*}, F(x)-F(\bar{x})-\left[-t T+(1+t) T^{\prime}\right](x-\bar{x})+\varepsilon\right\rangle \geq 0, \\
& \left\langle z_{1}^{*}, H(x)-H(\bar{x})-\left[-t L+(1+t) L^{\prime}\right](x-\bar{x})\right\rangle \geq 0 .
\end{aligned}
$$

Since $T \in \partial_{\frac{\epsilon}{2}} F(\bar{x})$ and $L \in \partial H(\bar{x})$, by using hypothesis of theorem, there exist $y^{*} \in K^{*} \backslash\{0\}^{2}$ and $z^{*} \in D^{*}$ such that

$$
\left\{\begin{array}{l}
y^{*} \circ(-T)+z^{*} \circ(-L) \in \partial\left(y^{*} \circ(-G)+z^{*} \circ(-S)\right)(\bar{x}) \\
\left\langle z^{*}, S(\bar{x})-H(\bar{x})\right\rangle=0 .
\end{array}\right.
$$

Consequently, for all $x \in X$ we get

$$
\left(y^{*} \circ(-G)+z^{*} \circ(-S)\right)(x)-\left(y^{*} \circ(-G)+z^{*} \circ(-S)\right)(\bar{x})-\left(y^{*} \circ(-T)+z^{*} \circ(-L)\right)(x-\bar{x}) \geq 0,
$$


which implies for all $x \in X$ that

$$
\left\langle y^{*},-G(x)+G(\bar{x})+T(x-\bar{x})\right\rangle+\left\langle z^{*},-S(x)+S(\bar{x})+L(x-\bar{x})\right\rangle \geq 0 .
$$

Since $y^{*} \in K^{*} \backslash\{0\} \subset K^{*}, z^{*} \in D^{*}$, it follows from (8) and the arbitrariness of $x$ on $U \cap \Omega$ that

$$
\begin{aligned}
& \left\langle y^{*}, F(x)-F(\bar{x})-\left[-t T+(1+t) T^{\prime}\right](x-\bar{x})+\epsilon\right\rangle \geq 0 \\
& \left\langle z^{*}, H(x)-H(\bar{x})-\left[-t L+(1+t) L^{\prime}\right](x-\bar{x})\right\rangle \geq 0 .
\end{aligned}
$$

Combining (9) and (10), we derive for all $x \in U \cap \Omega$

$$
\begin{aligned}
& \left\langle y^{*},(F(x)-G(x))-(F(\bar{x})-G(\bar{x}))+(1+t)\left(T-T^{\prime}\right)(x-\bar{x})+\epsilon\right\rangle \\
+ & \left\langle z^{*},(H(x)-S(x))-(H(\bar{x})-S(\bar{x}))+(1+t)\left(L-L^{\prime}\right)(x-\bar{x})\right\rangle \geq 0,
\end{aligned}
$$

That is,

$$
\begin{aligned}
& \left\langle y^{*},(F(x)-G(x))-(F(\bar{x})-G(\bar{x}))+\epsilon\right\rangle \\
+ & \left\langle z^{*},(H(x)-S(x))\right\rangle-\left\langle z^{*}, H(\bar{x})-S(\bar{x})\right\rangle \\
+ & \left\langle y^{*},(1+t)\left(T-T^{\prime}\right)(x-\bar{x})\right\rangle \\
+ & \left\langle z^{*},(1+t)\left(L-L^{\prime}\right)(x-\bar{x})\right\rangle \geq 0 .
\end{aligned}
$$

Hence since $H(x)-S(x) \in-D$, for all $x \in U \cap \Omega$ we get

$$
\left\langle z^{*}, H(x)-S(x)\right\rangle \leq 0 .
$$

Moreover, it can be deduced from $\left.\left\langle z^{*}, H(\bar{x})-S(\bar{x})\right)\right\rangle=0,(2)$ and (3) that

$$
\left\langle y^{*},(F(x)-G(x))-(F(\bar{x})-G(\bar{x}))+\epsilon\right\rangle+M(1+t) y^{*}(\alpha)+M(1+t) z^{*}(\gamma) \geq 0 .
$$

Since $\alpha$ and $\gamma$ are arbitrary elements respectively in $\operatorname{int} K$ and $\operatorname{int} D$, for all $n \in \mathbb{N}$ we have $\frac{\alpha}{n} \in i n t K$ and $\frac{\gamma}{n} \in \operatorname{int} D$. Therefore there exist neighborhood $U_{n}$ of $\bar{x}$ and sequence $\left\{t_{n}\right\}$ such that $t_{n} \rightarrow 0$ and for all $x \in U_{n} \cap \Omega$ we get,

$\left\langle y^{*},(F(x)-G(x))-(F(\bar{x}-G(\bar{x})))+\epsilon\right\rangle+M\left(1+t_{n}\right) y^{*}\left(\frac{\alpha}{n}\right)+M\left(1+t_{n}\right) z^{*}\left(\frac{\gamma}{n}\right) \geq 0$.

Now, assume that $V=\cup_{n=1}^{\infty} V_{n}$, where $V_{n}$ are the increasing sequences subset of $U_{n}$. Hence, by the latter inequality and choosing sufficiently large $n \in \mathbb{N}$, it can be deduced for all $x \in V \cap \Omega$

$\left\langle y^{*},(F(x)-G(x))-(F(\bar{x})-G(\bar{x}))+\epsilon\right\rangle+M \frac{\left(1+t_{n}\right)}{n} y^{*}(\alpha)+M \frac{\left(1+t_{n}\right)}{n} z^{*}(\gamma) \geq 0$, 
A. Shafie - Necessary and sufficient optimality conditions for ...

by letting $n \rightarrow+\infty$, we finally conclude that

$$
\left\langle y^{*},(F(x)-G(x))-(F(\bar{x})-G(\bar{x}))+\epsilon\right\rangle \geq 0 .
$$

Therefore, considering $K$ is a pointed cone

$$
(F(x)-G(x))-(F(\bar{x})-G(\bar{x}))+\epsilon \notin-i n t K \quad \forall x \in U \cap \Omega,
$$

which completes the proof of the theorem.

The following example shows that the boundedness of $\phi$ in Theorem (2.1) is essential.

Example 2.1. Let $X=\mathbb{R}, Y=\mathbb{R}^{2}, Z=\mathbb{R}, K=\mathbb{R}_{+}^{2}, D=\mathbb{R}_{+}, \epsilon=0, \bar{x}=0$. Now, we define the mappings $F, G: X \longrightarrow Y$ and $H, S: X \longrightarrow Z$ as follows:

$$
F(x)=\left(x^{2}, x^{2}\right), G(x)=(x, x), H(x)=x, S(x)=0, \phi(x, y)=|x-y| .
$$

We have $F, G: X \longrightarrow Y$ are $K$-convex and $H, S: X \longrightarrow Z$ are $D-$ convex and

$$
\partial F(x)=\{(2 x, 2 x)\}, \quad \partial G(x)=\{(1,1)\}, \partial S(x)=\{0\}, \partial H(x)=\{1\}
$$

and $\phi$ is unbounded. By Remark (2.1) $\partial H(x)$ is $\phi$-directional approximately pseudo dissipative and $\partial F(x)$ is $\phi$-directional approximately pseudo dissipative suppose that $x \in S_{X}=B(0,1), \epsilon=(a, b) \in \operatorname{int} K$ be given. Let $\delta=\min \left\{\frac{a}{[2(1+a)]}, \frac{b}{[2(1+b)]}\right\}$ so for all $x \in B(u, \delta), t \in(0, \delta)$ let $T^{*}=(2 t x .2 t x) \in \partial F(\bar{x}+t x)=\{(2 t x, 2 t x)\}$ and $T=(0,0) \in \partial F(\bar{x})=\{(0,0)\}$ so we have

$$
a|x| \geq 2 t x^{2}, b|x| \geq 2 t x^{2}
$$

or

$$
\left(T^{*}-T\right)(x-\bar{x})=(2 t x, 2 t x)_{(x)}=\left(2 t x^{2}, 2 t x^{2}\right) \leq_{K} \epsilon \phi\left(x, x^{-}\right)=(a|x|, b|x|) .
$$

Also for $T \in \partial_{\frac{\epsilon}{2}} F(\bar{x})=\{(0,0)\}, L \in \partial H(\bar{x})=\{1\}$ we consisder $y^{*}=(1,0), z^{*}=1$ so we have

$$
\left\{\begin{array}{l}
y^{*} \circ(-T)+z^{*} \circ(-L) \in \partial\left(y^{*} \circ(-G)+z^{*} \circ(-S)\right)(\bar{x}), \\
\left\langle z^{*}, H(\bar{x})-S(\bar{x})\right\rangle=0
\end{array}\right.
$$

but $\bar{x}$ is not an $\epsilon$-weak local Pareto minimal solution of problem $(P)$ because there is not neighbourhood $U$ of $\bar{x}=0$ such that

$$
(F(x)-G(x))-(F(\bar{x})-G(\bar{x}))+\epsilon=\left(x^{2}-x, x^{2}-x\right) \notin-\operatorname{int} K
$$


A. Shafie - Necessary and sufficient optimality conditions for ...

Remark 2.3. It is important to notice that if $\epsilon=0$, Theorem (2.1) provides sufficient optimality conditions for a weak pareto minimal solution of the vector optimization problem (P). The notion of Diff-Max maps which was first introduced by Michelot [2] means that each point of the effective domain is a local maximum for the subdifferential according to the inclusion relation, or from the $\epsilon$-subdifferential. In fact, $F$ is said to be Diff-Max at $\bar{x}$ iff there exists neiborhood of $\bar{x}$ such that for all $x \in U(\bar{x})$, the following inclusion holds

$$
\partial F(x) \subseteq \partial F(\bar{x})
$$

Note that if $F$ is Diff-Max at $\bar{x}$ then $\partial F$ is directionally approximately pseduodissipative at $\bar{x}$.

Remark 2.4. If $\epsilon=0$, then Theorem (2.1) is a sufficient optimality condition for a proper Pareto minimal solution for the vector optimization problem $(\mathrm{P})$.

\section{Necessary Optimality Conditions}

In this section we present a neccessary optimality conditions for D.C vector optimization problems. In order to prove the result we need the following lemma which is an improvement of the corresponding lemma given in [10] with a new and an easy proof.

Lemma 3.1. Let $C$ be a convex subset of $X$. If the map $F: C \longrightarrow Y$ is $K$-convexlike and $G: C \longrightarrow Z$ is $D$-convexlike and the system

$$
\left\{\begin{array}{l}
F(x) \in-i n t K \\
G(x) \in-i n t D
\end{array}\right\}
$$

has no solution in $C$, then there exist $\left(y^{*}, z^{*}\right) \in K^{*} \times D^{*}$ such that $\left(y^{*}, z^{*}\right) \neq$ $(0,0)$,

$$
\left\langle y^{*}, F(x)\right\rangle+\left\langle z^{*}, G(x)\right\rangle \geq 0 \quad \forall x \in C .
$$

Proof. We can easily prove that $F(C)+K$ and $G(C)+D$ are convex set. Let

$$
\begin{aligned}
& g: C \rightrightarrows 2^{X \times Y} \\
& g(x)=(F(x)+K) \times(G(x)+D)
\end{aligned}
$$

so we have $g(C) \cap \operatorname{int}(-K \times-D)=\emptyset$ and since $g(C)$ is convex set by the sepration theorem, there exists a nonzero $\left(y^{*}, z^{*}\right) \in\left(Y^{*}, Z^{*}\right)$ such that

$\left\langle y^{*},-k\right\rangle+\left\langle z^{*},-d\right\rangle \leq \alpha \leq\left\langle y^{*}, F(x)+k\right\rangle+\left\langle z^{*}, G(x)+d\right\rangle \quad$ for all $(k, d) \in(K, D), x \in C$ 
A. Shafie - Necessary and sufficient optimality conditions for ...

and this implies that $\left(y^{*}, z^{*}\right) \in\left(K^{*}, D^{*}\right)$ and

$$
\left\langle y^{*}, F(x)\right\rangle+\left\langle z^{*}, G(x)\right\rangle \geq 0 \quad \forall x \in C .
$$

Remark 3.1. One can verify, by slightly modifications of the proof of Lemma 3.1, that the result of Lemma 3.1 is still valid when

$$
\left\{\begin{array}{l}
F(x) \in-i n t K \\
G(x) \in-i n t D
\end{array}\right\}
$$

replace by

$$
\left\{\begin{array}{l}
F(x) \subseteq-i n t K \\
G(x) \subseteq-i n t D
\end{array}\right\}
$$

The following example shows that the Lemma 3.1 is a real improvement of the corresponding lemma appeared in [10].

Example 3.1. Let $X=\mathbb{R}, Y=\mathbb{R}^{2}, C=[0, \pi], K=D=\mathbb{R}_{+}^{2}, F(x)=(0, \sin x), G(x)=$ $(0, \cos x)$ therefore $F$ is $K$-convexlike and $G$ is $D$-convexlike but are not $K$-convex and $D$-convex, setting $x^{*}=(1,0), y^{*}=(1,0)$ we have

$$
\left\langle x^{*}, F(x)\right\rangle+\left\langle y^{*}, G(x)\right\rangle=0 \geq 0 .
$$

The following example shows that the seceond part of the result of the Theorem (4.1) of [12] may fault. This is the main reason why we omited it in the conclusion of the Theorem (4.1). In other words, Happing the parts of the result of the Theorem (4.1) of [12] simultaneously is impossible.

Example 3.2. Take $X=\mathbb{R}, Y=Z=\mathbb{R}^{2}, C=[-2,-1], K=D=[0,+\infty) \times$ $[0,+\infty), \bar{x}=\circ, \varepsilon=(\circ, \circ)$. Consider $F, G, H, S: \mathbb{R} \rightarrow \mathbb{R}^{2}$ defined by

$$
\left\{\begin{array}{l}
F(x)=(x, 0) \\
G(x)=(2 x, 0) \\
H(x)=(2 x-1,-1) \\
S(x)=\left(x^{2}, 0\right)
\end{array}\right.
$$

Clearly $F, G$ are $K$-convex and $H, S$ are $D$-convex, with $\partial G(\bar{x})=\{2\}, \partial H(\bar{x})=$ $\{2\}$. So

$$
F(x)-G(x)-(F(\bar{x})-G(\bar{x}))=(-x, 0) \notin-\operatorname{int} K, \quad \forall x \in U(0) \cap C
$$


A. Shafie - Necessary and sufficient optimality conditions for ...

which implies that $\bar{x}=0$ is $\varepsilon$-weak local minimal solution of $(P)$, Now if

$$
\left\langle z^{*}, H(\bar{x})-S(\bar{x})\right\rangle=\left\langle z^{*},(-1,-1)\right\rangle=0 \Rightarrow z^{*}=0
$$

and

$$
\left(y^{*} \circ \partial G+z^{*} \circ \partial H\right)=2 y^{*} \in \partial\left(y^{*} o(x, 0)+\delta_{U \cap \Omega}\right)_{(\bar{x})}
$$

which implies that $x \leq 0$ for all $x \in U \cap C$ which is contradiction.

The above example is also a counterexample for Theorem [4.1,4.2] of [?]. So corallaris $[4.2,4.3,4.4,4.5,4.6]$ are not correct.

The following Theorem modify and extend the Theorem (4.1) of [12].

Theorem 3.2. Let $\bar{x} \in \Omega$. If the vector-valued map $F: X \longrightarrow Y$ is a $K$-convexlike map, the vector-valued map $H: X \longrightarrow Z$ is a D-convexlike map, and $\bar{x}$ is an $\epsilon$-weak local minimal solution of $(P)$, then there exist $\left(y^{*}, z^{*}\right) \in K^{*} \times D^{*}$ and $\left(y^{*}, z^{*}\right) \neq\left(0_{y^{*}}, 0_{z^{*}}\right)$ such that

$$
\left(y^{*} \circ \partial G+z^{*} \circ \partial H\right)(\bar{x}) \subset \partial_{\left\langle y^{*}, \epsilon\right\rangle}\left(y^{*} \circ F+z^{*} \circ H+\delta_{U \cap C}\right)(\bar{x}) .
$$

Proof. Let $\bar{x} \in \Omega$ and $\epsilon \in K$. Since $\bar{x}$ is an $\epsilon$-weak local minimal solution of (P), there exists a neighborhood $U$ of $\bar{x}$ such that for all $x \in U \cap C$,

$$
F(x)-G(x)-(F(\bar{x})-G(\bar{x}))+\epsilon \notin-i n t K .
$$

Now suppose that $T \in \partial G(\bar{x})$ and $L \in \partial H(\bar{x})$ be an arbitrary elements. Since $F: X \longrightarrow Y$ is a $K$-convexlike and $G: X \longrightarrow Z$ is a $D$-convexlike maps, therefore it is easy to check that $F()-.F(\bar{x})-T(.-\bar{x})+\epsilon$ is a $K$-convexlike map and $H()-.H(\bar{x})-L(.-\bar{x})$ is a $D$-convexlike map. Using the latter, we prove that the system

$$
\left\{\begin{array}{l}
F(x)-F(\bar{x})-T(x-\bar{x})+\epsilon \in-\operatorname{int} K \\
H(x)-H(\bar{x})-L(x-\bar{x}) \in-\operatorname{int} D,
\end{array}\right.
$$

has no solution in $U \cap C$. Arguing by contradiction, assume that there exists a solution $x_{0} \in U \cap C$ of (11). Thus

$$
\left\{\begin{array}{l}
F\left(x_{0}\right)-F(\bar{x})-T\left(x_{0}-\bar{x}\right)+\epsilon \in-i n t K, \\
H\left(x_{0}\right)-H(\bar{x})-L\left(x_{0}-\bar{x}\right) \in-i n t D .
\end{array}\right.
$$

Since $T \in \partial G(\bar{x})$ and $L \in \partial S(\bar{x})$, we have

$$
G(x)-G(\bar{x})-T(x-\bar{x}) \in K \quad \forall x \in X,
$$


A. Shafie - Necessary and sufficient optimality conditions for ...

and

$$
S(x)-S(\bar{x})-L(x-\bar{x}) \in D \quad \forall x \in X .
$$

Let $x=x_{0}$, so we have

$$
\left\{\begin{array}{l}
-G\left(x_{0}\right)+G(\bar{x})+T\left(x_{0}-\bar{x}\right) \in-K, \\
-S\left(x_{0}\right)+S(\bar{x})+L\left(x_{0}-\bar{x}\right) \in-D .
\end{array}\right.
$$

Since $-K-$ int $K=-i n t K$ and $-D-i n t D=-i n t D$, and $H(\bar{x})-S(\bar{x}) \in-D$ combining (13) and (14) we obtain

$$
\left\{\begin{array}{l}
F\left(x_{0}\right)-G\left(x_{0}\right)-(F(\bar{x})-G(\bar{x}))+\epsilon \in-i n t K, \\
H\left(x_{0}\right)-S\left(x_{0}\right) \in-i n t D
\end{array}\right.
$$

this contradicts to the assumption that $\bar{x}$ is an $\epsilon$-weak local minimal solution of (P). Hence, system (12) has no solution. It follows from Lemma (3.1) that there exists $\left(y^{*}, z^{*}\right) \neq(0,0)$ such that for all $x \in U \cap C$

$$
\left\langle y^{*}, F(x)-F(\bar{x})-T(x-\bar{x})+\epsilon\right\rangle+\left\langle z^{*}, G(x)-G(\bar{x})-L(x-\bar{x})\right\rangle \geq 0
$$

Consequently,

$\left(y^{*} \circ F+z^{*} \circ H\right)(x)-\left(y^{*} \circ F+z^{*} \circ H\right)(\bar{x})+\left\langle y^{*}, \epsilon\right\rangle-\left(y^{*} \circ T+z^{*} \circ L\right)(x-\bar{x}) \geq 0$

Thus we have that,

$$
\left(y^{*} \circ \partial G+z^{*} \circ \partial H\right) \subset \partial_{\left\langle y^{*}, \epsilon\right\rangle}\left(y^{*} \circ F+z^{*} \circ H+\delta_{U \cap C}\right) .
$$

This completes the proof.

\section{REFERENCES}

[1] T.Amahroq, J.P.Penot, A.Syam, On the subdifferentiability of the difference of two functions and local minimization, Set-Valued Anal. 4 (2008),413-427.

[2] C.Michelot Caractrisation des minima locaux des fonctions de la classe D.C, Universit de Dijon (1987).

[3] J.P.Penot, Gap continuity of multimaps, Set-Valued Anal. 16 (4) (2008),429442 .

[4] J.P.Penot, The directional subdifferential of the difference of two convex functions, J. Glob. Optim. 49 (2011),505-519. 
A. Shafie - Necessary and sufficient optimality conditions for ...

[5] X.L.Guo, S.J.Li, Optimality conditions for vector optimization problems with difference of convex maps, J. Optim. Theory Appl. 162 (2014),821-844.

[6] N.Gadhi, M.Laghdir, A.Metrane, Optimality conditions for D.C. vector optimization problems under reverse convex constraints, J. Glob. Optim. 33 (2005),527540 .

[7] M.E.Maghri, M.Laghdir, Pareto subdifferential calculus for convex vector mappings and applications to vector optimization, SIAM J. Optim. 19 (2009),1970-1994.

[8] V.Pareto, Manuale di economia politica (Societa Editrice Libraria, Milano, Italy, 1906), English translation, V.Pareto, Manual of political economy, translated by A.S.Schwier, M.Augustus, Kelley Publishers, New York (1971).

[9] J.P.Penot, Gap continuity of multimaps, Set-Valued Anal. 16(4) (2008),429-442.

[10] Lin, L.J, Optimization of set-valued functions. J. Math. Anal. Appl. 186 (1994),30-51.

[11] N.Gadhi, Optimality conditions for the difference of convex set-valued mappings. Positivity. 94(2005),687-703.

[12] R. Bala, B. Ram, Trigonometric series with semi-convex coefficients, Tamang J. Math. 18, 1 (1987), 75-84.

[13] B. Ram, Convergence of certain cosine sums in the metric space L, Proc. Amer. Math. Soc. 66 (1977), 258-260.

Allahkaram Shafie

Department of Mathematics, Faculty of Science, University of Razi,

Kermanshah, Iran

email: shafie.allahkaram@gmail.com 